\title{
ON INTEGRALS OF EIGENFUNCTIONS OVER GEODESICS
}

\author{
XUEHUA CHEN AND CHRISTOPHER D. SOGGE
}

\begin{abstract}
If $(M, g)$ is a compact Riemannian surface then the integrals of $L^{2}(M)$ normalized eigenfunctions $e_{j}$ over geodesic segments of fixed length are uniformly bounded. Also, if $(M, g)$ has negative curvature and $\gamma(t)$ is a geodesic parameterized by arc length, the measures $e_{j}(\gamma(t)) d t$ on $\mathbb{R}$ tend to zero in the sense of distributions as the eigenvalue $\lambda_{j} \rightarrow \infty$, and so integrals of eigenfunctions over periodic geodesics tend to zero as $\lambda_{j} \rightarrow \infty$. The assumption of negative curvature is necessary for the latter result.
\end{abstract}

\section{General results.}

If $M$ is a compact hyperbolic surface Good [6] and Hejhal [7, using Kuznecov formulae, showed that if $\gamma$ is a periodic geodesic and $d s$ is the associated arc length measure then

$$
\left|\int_{\gamma} e_{\lambda} d s\right| \leq C_{\gamma}
$$

with $e_{\lambda}$ denoting the $L^{2}$-normalized eigenfunctions on $M$, i.e., $-\Delta_{g} e_{\lambda}=\lambda^{2} e_{\lambda}$, and $\left\|e_{\lambda}\right\|_{L^{2}(M)}=1$.

This result was generalized by Zelditch [14 who showed, among many other things, that given any $n$-dimensional compact Riemannian manifold, one has uniform bounds for integrals of eigenfunctions over closed hypersurfaces 1 Moreover, if $\lambda_{j}$ are the eigenvalues of $\sqrt{-\Delta_{g}}$ and $a_{j}(\gamma)$ denotes the integral in (1.1) with $\lambda=\lambda_{j}$, then [14, Lemma 3.1] says that $\sum_{\lambda_{j} \leq \lambda}\left|a_{j}(\gamma)\right|^{2}=c_{\gamma} \lambda+O(1)$, which implies (1.1). Note that since, by the Weyl law $\#\left\{j: \lambda_{j} \leq \lambda\right\} \approx \lambda^{2}$, Zelditch's formula says that most of the $a_{j}(\gamma)$ are much smaller than 1 .

Reznikov [8] discussed this problem and, moreover, initiated work on the related problem of obtaining restriction estimates for geodesics. The sharp $L^{2}$ estimates for general Riemannian surfaces were obtained by Burq, Gérard and Tzvetkov [3. If $\Pi$ denotes the space of unit length geodesics in a two-dimensional compact Riemannian manifold, then one of the results in [3] is that we have bounds of the form

$$
\left(\int_{\gamma}\left|e_{\lambda}\right|^{2} d s\right)^{1 / 2} \lesssim \lambda^{\frac{1}{4}}\left\|e_{\lambda}\right\|_{L^{2}(M)}, \quad \gamma \in \Pi .
$$

This estimate is sharp since it is saturated by the highest weight spherical harmonics on $S^{2}$. Recently, improvements under the assumption of nonpositive curvature have been obtained by Sogge and Zelditch 13 and Chen and Sogge 4 . Work showing how these restriction estimates are related to $L^{p}(M)$ estimates for eigenfunctions is in Bourgain [2] and Sogge [1].

The authors were supported in part by the NSF grant DMS-1069175 and the Simons Foundation.

${ }^{1}$ We are grateful to Steve Zelditch for helpful comments and referring us to the work based on the Kuznecov formula. 
Returning to (1.1), note that the estimate cannot be improved when the compact hyperbolic surface $M$ is replaced by the standard two-sphere $S^{2}$ or two-torus $\mathbb{T}^{2}$. For on $S^{2}$ zonal functions of even order saturate the bound, while for every periodic geodesic on $\mathbb{T}^{2}$ one can find a sequence of eigenvalues $\lambda_{j} \rightarrow \infty$ and corresponding $L^{2}$-normalized eigenfunctions $e_{\lambda_{j}}$ having the property that $e_{\lambda_{j}} \equiv 1$ on $\gamma$.

We shall start by giving a quick proof of a result in [14] saying that we have the analog of (1.1) for all geodesic segments in any Riemannian surface. The proof will serve as a template for the improvements in the next section of the bounds in (1.1) for Riemannian surfaces of negative curvature which appear to be new.

Theorem 1.1. Let $(M, g)$ be a two-dimensional compact Riemannian manifold. Then there is a constant $C=C(M, g)$ so that

$$
\left|\int_{\gamma} e_{\lambda} d s\right| \leq C\left\|e_{\lambda}\right\|_{L^{2}(M)}, \quad \gamma \in \Pi
$$

Proof. Fix an even function $\rho \in \mathcal{S}(\mathbb{R})$ satisfying $\rho(0)=1$ and $\hat{\rho}(t)=0,|t| \geq 1 / 4$, assuming, as we may, that the injectivity radius of $(M, g)$ is ten or more. Then since $\left.\rho\left(\lambda-\sqrt{-\Delta_{g}}\right)\right) e_{\lambda}=e_{\lambda}$, in order to prove (1.2), it suffices to show that

$$
\left|\int_{\gamma} \rho\left(\lambda-\sqrt{-\Delta_{g}}\right) f d s\right| \leq C\|f\|_{L^{2}(M)}, \quad \gamma \in \Pi
$$

Let $\gamma(t), 0 \leq t \leq 1$, be a parameterization of $\gamma$ by arc length and

$$
\rho\left(\lambda-\sqrt{-\Delta_{g}}\right)(x, y)=\sum \rho\left(\lambda-\lambda_{j}\right) e_{j}(x) \overline{e_{j}(y)}
$$

denote the kernel of the operator in (1.4). Here, $\left\{e_{j}\right\}$ is an orthonormal basis of eigenfunctions with eigenvalues $\left\{\lambda_{j}\right\}$.

By Schwarz's inequality, we would have (1.4) if we could show that

$$
\int_{M}\left|\int_{0}^{1} \sum_{j} \rho\left(\lambda-\lambda_{j}\right) e_{j}(\gamma(t)) \overline{e_{j}(y)} d t\right|^{2} d V_{g}(y) \leq C
$$

By orthogonality, if $\chi(\tau)=(\rho(\tau))^{2}$, this is equivalent to showing that

$$
\left|\int_{0}^{1} \int_{0}^{1} \sum_{j} \chi\left(\lambda-\lambda_{j}\right) e_{j}(\gamma(t)) \overline{e_{j}(\gamma(s))} d t d s\right| \leq C .
$$

Next, we note that the proof of [10, Lemma 5.1.3] shows that if $d_{g}$ denotes the Riemannian distance then we can write

$$
\sum_{j} \chi\left(\lambda-\lambda_{j}\right) e_{j}(x) \overline{e_{j}(y)}=\lambda^{\frac{1}{2}} \sum_{ \pm} a_{ \pm}\left(\lambda ; d_{g}(x, y)\right) e^{ \pm i \lambda d_{g}(x, y)}+O(1)
$$

where for every fixed $j=0,1,2, \ldots$ we have the uniform bounds

$$
\left|\frac{d^{j}}{d r^{j}} a_{ \pm}(\lambda ; r)\right| \leq C_{j} r^{-j-\frac{1}{2}}, \quad \text { if } r \geq \lambda^{-1}
$$

and

$$
\left|a_{ \pm}(\lambda, r)\right| \leq \lambda^{\frac{1}{2}}, \quad \text { if } r \in\left[0, \lambda^{-1}\right]
$$

To obtain (1.6)-(1.8), as in [10, §5.1] one uses Hörmander's parametrix for the half-wave operators $e^{i t \sqrt{-\Delta_{g}}}$, as well as the fact that $\hat{\chi}(t)=0$ for $|t|>1$ and our assumption that the injectivity radius of $(M, g)$ is ten or more. 
Since $d_{g}(\gamma(t), \gamma(s))=|t-s|$, we conclude that we would have (1.5) if

$$
\lambda^{\frac{1}{2}}\left|\int_{0}^{1} \int_{0}^{1} e^{ \pm i \lambda|t-s|} a_{ \pm}(\lambda ;|t-s|) d t d s\right| \leq C .
$$

Since this is a trivial consequence of (1.7) and (1.8), the proof is complete.

Reznikov in 8 and [9] also discusses the problem of integrals of eigenfunctions over geodesics circles in compact hyperbolic surfaces and obtains the analog of (1.1) for them. More general results were obtained earlier by Zelditch [14, Corollary 3.3], and the proof of Theorem 1.1 can also be used to obtain these special cases of the latter:

Theorem 1.2. Let $(M, g)$ be a compact two-dimensional Riemannian manifold. Then if $\sigma$ is a unit-length curve in $M$ there is a constant $C_{\sigma}$ so that

$$
\left|\int_{\sigma} e_{\lambda} d s\right| \leq C_{\sigma}\left\|e_{\lambda}\right\|_{L^{2}(M)} .
$$

Similar bounds with a uniform constant hold for small smooth perturbations of $\sigma$.

\section{Improved results for negative curvature.}

We conclude our note by showing that we can improve the bounds in (1.1) if we assume that the curvature of $(M, g)$ is strictly negative. As noted before, this assumption is necessary since the corresponding result is false for the two-sphere and the two-torus. We shall now assume that $\gamma(t), t \in \mathbb{R}$, is a geodesic in $\mathbb{R}$ parameterized by arc length, and our main result is the following

Theorem 2.1. Let $(M, g)$ be a negatively curved compact 2-dimensional Riemannian manifold. Then the measures $\left\{e_{j}(\gamma(t)) d t\right\}$ on $\mathbb{R}$ go to zero in the sense of distributions, by which we mean that if $b \in C_{0}^{\infty}(\mathbb{R})$ then

$$
\int b(t) e_{j}(\gamma(t)) d t \rightarrow 0, \quad \text { as } j \rightarrow \infty
$$

Consequently, if $\gamma_{\text {per }}$ is a periodic geodesic of minimal period $\ell>0$, we have

$$
\int_{0}^{\ell} e_{j}\left(\gamma_{\text {per }}(t)\right) d t \rightarrow 0, \quad \text { as } j \rightarrow \infty .
$$

The second part of the lemma follows from the first part via a partition of unity argument. The proof of (2.1) shares some similarities with the related $L^{p}(\gamma)$ restriction estimates for eigenfunctions of Sogge and Zelditch 13 and Chen and Sogge [4. In particular, the oscillatory integral arguments and simple geometric facts that we shall employ are very similar to those in 44.

To prove (2.1) we may assume that the injectivity radius of $(M, g)$ is ten or more and that

$$
\text { supp } b \subset\left[-\frac{1}{2}, \frac{1}{2}\right] \text {. }
$$

Next, we notice that if, as before, $\rho \in \mathcal{S}(\mathbb{R})$ is even and satisfies $\rho(0)=1$ and $\hat{\rho}(t)=0$, $|t| \geq 1 / 4$, then given $T \gg 1$ we have $\left.\rho\left(T\left(\lambda-\sqrt{-\Delta_{g}}\right)\right)\right) e_{\lambda}=e_{\lambda}$. As a result, in order to prove (2.1) it suffices to verify that if $T \gg 1$ then

$$
\left|\int b(s)\left(\rho\left(T\left(\lambda-\sqrt{-\Delta_{g}}\right)\right) f\right)(\gamma(s)) d s\right| \leq\left(C T^{-\frac{1}{2}}+C_{T} \lambda^{-\frac{1}{4}}\right)\|f\|_{L^{2}(M)},
$$


where $C$ is independent of $T \gg 1$, but not $C_{T}$. Repeating the argument which showed how (1.5) implies (1.4), we conclude that if $\chi(\tau)=(\rho(\tau))^{2}$ and

$$
b(t, s)=b(t) b(s),
$$

then we would have (2.2) if we could show that

$$
\mid \iint b(t, s) \sum_{j} \chi\left(T\left(\lambda-\lambda_{j}\right)\right) e_{j}\left(\gamma(t) \overline{e_{j}(\gamma(s))} d t d s \mid \leq C T^{-1}+C_{T} \lambda^{-\frac{1}{2}} .\right.
$$

Note that

$$
\sum_{j} \chi\left(T\left(\lambda-\lambda_{j}\right)\right) e_{j}(x) \overline{e_{j}(y)}=\frac{1}{2 \pi T} \int \hat{\chi}(\tau / T) e^{-i \tau \lambda} e^{i \tau \sqrt{-\Delta_{g}}} d \tau .
$$

If we pick a bump function $\beta \in C_{0}^{\infty}(\mathbb{R})$ satisfying

$$
\beta(\tau)=1, \quad|\tau| \leq 3, \text { and } \beta(\tau)=0,|\tau| \geq 4,
$$

the proof of (1.5) shows that if $\Psi_{T}$ denotes the inverse Fourier transform of $\tau \rightarrow$ $\beta(\tau) \chi(\tau / T)$, then

$$
\begin{aligned}
\left|\frac{1}{2 \pi T} \iiint b(t, s) \beta(\tau) \hat{\chi}(\tau / T) e^{-i \tau \lambda}\left(e^{i \tau \sqrt{-\Delta_{g}}}\right)(\gamma(t), \gamma(s)) d \tau d t d s\right| \\
=T^{-1}\left|\iint b(t, s) \sum_{j} \Psi_{T}\left(\lambda-\lambda_{j}\right) e_{j}(\gamma(t)) \overline{e_{j}(\gamma(s))} d t d s\right| \leq C T^{-1} .
\end{aligned}
$$

Here, $\left(e^{i \tau \sqrt{-\Delta_{g}}}\right)(x, y)=\sum_{j} e^{i \tau \lambda_{j}} e_{j}(x) \overline{e_{j}(y)}$ denotes the kernel of the half-wave operator $e^{i \tau} \sqrt{-\Delta_{g}}$.

Based on the preceding inequality, in order to prove (2.3), it suffices to show that

$$
\begin{aligned}
\left|\frac{1}{2 \pi} \iiint b(t, s)(1-\beta(\tau)) \hat{\chi}(\tau / T) e^{-i \tau \lambda}\left(e^{i \tau \sqrt{-\Delta_{g}}}\right)(\gamma(t), \gamma(s)) d \tau d t d s\right| & \\
& \leq 1+C_{T} \lambda^{-\frac{1}{2}}
\end{aligned}
$$

We shall need to use the fact that since $\hat{\chi}=(2 \pi)^{-1} \hat{\rho} * \hat{\rho}$, we have

$$
\hat{\chi}(\tau)=0, \quad|\tau| \geq \frac{1}{2},
$$

which means that the $\tau$ integrand in the left side of (2.4) vanishes when $|\tau| \geq T / 4$. We can make one more easy reduction. If $\Phi_{T}$ denotes the inverse Fourier transform of $\tau \rightarrow(1-\beta(\tau)) \chi(\tau / T)$ then $\Phi_{T} \in \mathcal{S}(\mathbb{R})$ and consequently

$$
\sum_{j} \Phi_{T}\left(\lambda+\lambda_{j}\right) e_{j}(\gamma(t)) \overline{e_{j}(\gamma(s))}=O_{T, N}\left((1+\lambda)^{-N}\right)
$$

for any $N=1,2,3, \ldots$ Thus, by Euler's formula and (2.5), in order to prove (2.4), it suffices to show that

$$
\begin{array}{r}
\left|\iiint_{-T / 2}^{T / 2} b(t, s)(1-\beta(\tau)) \hat{\chi}(\tau / T) e^{-i \tau \lambda}\left(\cos \tau \sqrt{-\Delta_{g}}\right)(\gamma(t), \gamma(s)) d \tau d t d s\right| \\
\leq 1+C_{T} \lambda^{-\frac{1}{2}}
\end{array}
$$


Here $\left(\cos \tau \sqrt{-\Delta_{g}}\right)(x, y)$ is the kernel for the map $C^{\infty}(M) \ni f \rightarrow u \in C^{\infty}(\mathbb{R} \times M)$, where $u(t, x)$ is the solution of the Cauchy problem with initial data $(f, 0)$, i.e.

$$
\left(\partial_{t}^{2}-\Delta_{g}\right) u=0, u(0, \cdot)=f, \partial_{t} u(0, \cdot)=0 .
$$

To be able to compute the integral in (2.6) we need to relate this wave kernel to the corresponding one in the universal cover for $(M, g)$. Recall that by a theorem of Hadamard (see [5, Chapter 7]) for every point $P \in M$, the exponential map at $P, \exp _{P}: T_{P} M \rightarrow M$ is a covering map. We might as well take $P=\gamma(0)$ to be the midpoint of the geodesic segment $\left\{\gamma(t):|t| \leq \frac{1}{2}\right\}$. If we identify $T_{P} M$ with $\mathbb{R}^{2}$, and let $\kappa$ denote this exponential map then $\kappa: \mathbb{R}^{2} \rightarrow M$ is a covering map. We also will denote by $\tilde{g}$ the metric on $\mathbb{R}^{2}$ which is the pullback via $\kappa$ of the the metric $g$ on $M$. Also, let $\Gamma$ denote the group of deck transformations, which are the diffeomorphisms $\alpha$ from $\mathbb{R}^{2}$ to itself preserving $\kappa$, i.e., $\kappa=\kappa \circ \alpha$. Next, let

$$
D_{\text {Dir }}=\left\{\tilde{y} \in \mathbb{R}^{2}: d_{\tilde{g}}(0, \tilde{y})<d_{\tilde{g}}(0, \alpha(\tilde{y})), \forall \alpha \in \Gamma, \alpha \neq \text { Identity }\right\}
$$

be the Dirichlet domain for $\left(\mathbb{R}^{2}, \tilde{g}\right)$, where $d_{\tilde{g}}(\cdot, \cdot)$ denotes the Riemannian distance function for $\mathbb{R}^{2}$ corresponding to the metric $\tilde{g}$. We can then add to $D_{D i r}$ a subset of $\partial D_{\text {Dir }}=\overline{D_{\text {Dir }}} \backslash$ Int $\left(D_{\text {Dir }}\right)$ to obtain a natural fundamental domain $D$, which has the property that $\mathbb{R}^{2}$ is the disjoint union of the $\alpha(D)$ as $\alpha$ ranges over $\Gamma$ and $\left\{\tilde{y} \in \mathbb{R}^{2}\right.$ : $\left.d_{\tilde{g}}(0, \tilde{y})<10\right\} \subset D$ since we are assuming that the injectivity radius of $(M, g)$ is more than ten. It then follows that we can identify every point $x \in M$ with the unique point $\tilde{x} \in D$ having the property that $\kappa(\tilde{x})=x$. Let also $\tilde{\gamma}(t),|t| \leq \frac{1}{2}$ similarly denote those points in $D$ corresponding to our geodesic segment $\gamma(t),|t| \leq \frac{1}{2}$ in $M$. Then $\left\{\tilde{\gamma}(t):|t| \leq \frac{1}{2}\right\}$ is a line segment of unit length whose midpoint is the origin, and we shall denote just by $\tilde{\gamma}$ the line through the origin containing this segment. Note that $\tilde{\gamma}$ then is a geodesic in $\mathbb{R}^{2}$ for the metric $\tilde{g}$, and the Riemannian distance between two points on $\tilde{\gamma}$ agrees with their Euclidean distance. Finally, if $\Delta_{\tilde{g}}$ denotes the Laplace-Beltrami operator associated to $\tilde{g}$ then since solutions of the Cauchy problem (2.7) correspond exactly to periodic (i.e. $\Gamma$-invariant) solutions of the corresponding Cauchy problem associated to $\partial_{t}^{2}-\Delta_{\tilde{g}}$, we have the following important formula relating the wave kernel on $(M, g)$ to the one for the universal cover $\left(\mathbb{R}^{2}, \tilde{g}\right)$ :

$$
\left(\cos \tau \sqrt{-\Delta_{g}}\right)(x, y)=\sum_{\alpha \in \Gamma}\left(\cos \tau \sqrt{\Delta_{\tilde{g}}}\right)(\tilde{x}, \alpha(\tilde{y}))
$$

The simple geometric facts that we require is in the following variation of [4, Lemma 3.2]:

Lemma 2.2. Let $\tilde{\gamma}_{1}(t)$ and $\tilde{\gamma}_{2}(s)$ be two distinct geodesics in $\left(\mathbb{R}^{2}, \tilde{g}\right)$ each parameterized by arc length. Put

$$
\phi(t, s)=d_{\tilde{g}}\left(\tilde{\gamma}_{1}(t), \tilde{\gamma}_{2}(s)\right) .
$$

Then if there is a point $\left(t_{0}, s_{0}\right) \in \mathbb{R} \times \mathbb{R}$ such that $\partial_{t} \phi\left(t_{0}, s_{0}\right)=\partial_{s} \phi\left(t_{0}, s_{0}\right)=0$ and $\gamma_{1}\left(t_{0}\right) \neq \gamma_{2}\left(s_{0}\right)$, then

$$
\left|\partial_{s} \phi(t, s)\right|+\left|\partial_{t} \phi(t, s)\right| \neq 0 \quad \text { if } \tilde{\gamma}_{1}(t) \neq \tilde{\gamma}_{2}(s) \text { and }(t, s) \neq\left(t_{0}, s_{0}\right) \text {, }
$$

and

$$
\partial_{t} \partial_{s} \phi\left(t_{0}, s_{0}\right) \neq 0
$$

Proof. To prove (2.9) we first note that if $\tilde{\gamma}_{1}\left(t_{0}\right) \neq \tilde{\gamma}_{2}\left(s_{0}\right)$ then $\left|\partial_{t} \phi\left(t_{0}, s_{0}\right)\right|+\left|\partial_{s} \phi\left(t_{0}, s_{0}\right)\right|=$ 0 if and only if the geodesic connecting the points $\tilde{\gamma}_{1}\left(t_{0}\right)$ and $\tilde{\gamma}_{2}\left(s_{0}\right)$ is perpendicular to both $\tilde{\gamma}_{1}$ and $\tilde{\gamma}_{2}$ at the unique intersection points. Since $\left(\mathbb{R}^{2}, \tilde{g}\right)$ has negative curvature 
there cannot be another point $\left(t_{1}, s_{1}\right) \in \mathbb{R} \times \mathbb{R}$ with this property. For if $t_{0} \neq t_{1}$ and $s_{0} \neq s_{1}$ the geodesic quadrilateral with vertices $\tilde{\gamma}_{1}\left(t_{0}\right), \tilde{\gamma}_{1}\left(t_{1}\right), \tilde{\gamma}_{2}\left(s_{0}\right)$ and $\tilde{\gamma}_{2}\left(s_{1}\right)$ would have total angle $2 \pi$, which is impossible due to the fact that $\left(\mathbb{R}^{2}, \tilde{g}\right)$ is negatively curved, and similarly if $t_{0}=t_{1}$ but $s_{0} \neq s_{1}$ then the geodesic triangle with vertices $\tilde{\gamma}_{1}\left(t_{0}\right), \tilde{\gamma}_{2}\left(s_{0}\right)$ and $\tilde{\gamma}_{2}\left(s_{1}\right)$ would have total angle of more than $\pi$, which is also impossible.

To prove (2.10) we may assume that $t_{0}=0$ and work in geodesic normal coordinates vanishing at $\gamma_{1}(0)$ so that $\gamma_{1}$ is the $x_{1}$-axis, i.e., $\gamma_{1}(t)=(t, 0)$. Then if $\gamma_{2}(s)=$ $\left(x_{1}(s), x_{2}(s)\right) \neq(0,0)$,

$$
\frac{\partial \phi}{\partial t}(0, s)=\frac{-x_{1}(s)}{\sqrt{x_{1}^{2}(s)+x_{2}^{2}(s)}} .
$$

Our assumption that $\frac{\partial \phi}{\partial t}\left(0, s_{0}\right)=0$ and $\gamma_{1}(0) \neq \gamma_{2}\left(s_{0}\right)$ means that $x_{1}\left(s_{0}\right)=0$ and $x_{2}\left(s_{0}\right) \neq 0$. In our coordinates the geodesic connecting $(0,0)=\gamma_{1}(0)$ and $\gamma_{2}\left(s_{0}\right)$ is the $x_{2}$-axis, and for it to be orthogonal to $\gamma_{2}$ at $\gamma_{2}\left(s_{0}\right)$, we must have $x_{2}^{\prime}\left(s_{0}\right)=0$ and so $x_{1}^{\prime}\left(s_{0}\right) \neq 0$. But then

$$
\frac{\partial^{2} \phi}{\partial s \partial t}\left(0, s_{0}\right)=-\frac{x_{1}^{\prime}\left(s_{0}\right)}{\left|x_{2}\left(s_{0}\right)\right|} \neq 0,
$$

which is (2.10).

We also need the following simple stationary phase lemma.

Lemma 2.3. Let $a \in C_{0}^{\infty}\left(\mathbb{R}^{2}\right)$ and assume that $\phi \in C^{\infty}\left(\mathbb{R}^{2}\right)$ is real. Put

$$
I(\lambda)=\iint e^{i \lambda \phi(t, s)} a(t, s) d t d s, \quad \lambda \geq 1 .
$$

Then

$$
|I(\lambda)| \leq C \lambda^{-1} \text { if } \nabla_{t, s} \phi(t, s) \neq 0,(t, s) \in \operatorname{supp} a .
$$

Also, if there is a unique point $\left(t_{0}, s_{0}\right) \in \operatorname{supp}$ a at which $\nabla_{t, s} \phi\left(t_{0}, s_{0}\right)=0$ and if $\frac{\partial^{2} \phi}{\partial t \partial s}\left(t_{0}, s_{0}\right) \neq 0$ then given any $\varepsilon>0$ there is a constant $C_{\varepsilon}$ so that

$$
|I(\lambda)| \leq \varepsilon \lambda^{-\frac{1}{2}}+C_{\varepsilon} \lambda^{-1} .
$$

Proof. The first assertion, (2.11), just follows via integration by parts. To prove (2.12) we assume that there is a $\left(t_{0}, s_{0}\right) \in \operatorname{supp} a$ at which $\nabla \phi$ vanishes but $\nabla \phi(t, s) \neq 0$, $(t, s) \in \operatorname{supp} a \backslash\left\{\left(t_{0}, s_{0}\right)\right\}$ and $\partial_{t} \partial_{s} \phi\left(t_{0}, s_{0}\right) \neq 0$. We can split matters into two further cases: (i) $\partial_{t}^{2} \phi\left(t_{0}, s_{0}\right)=0$ and (ii) $\partial_{t}^{2} \phi\left(t_{0}, s_{0}\right) \neq 0$.

In case (i), we note that our assumptions mean that at $\left(t_{0}, s_{0}\right)$ the mixed Hessian of $\phi$ satisfies

$$
\operatorname{det}\left(\begin{array}{cc}
\phi_{t t}^{\prime \prime} & \phi_{t s}^{\prime \prime} \\
\phi_{t s}^{\prime \prime} & \phi_{s s}^{\prime \prime}
\end{array}\right) \neq 0
$$

and, therefore, by two-dimensional stationary phase we have $|I(\lambda)| \leq C \lambda^{-1}$.

To finish, it suffices to show that we have (2.12) under the assumption that

$$
\nabla \phi(t, s) \neq 0, \quad(t, s) \in \operatorname{supp} a \backslash\left\{\left(t_{0}, s_{0}\right)\right\}, \text { and } \partial_{t}^{2} \phi\left(t_{0}, s_{0}\right) \neq 0 .
$$

If we let $\beta$ be as above, it follows from (2.11) that given any fixed $\varepsilon>0$, we have

$$
I_{1}(\lambda)=\iint e^{i \lambda \phi(t, s)}\left(1-\beta\left(\varepsilon^{-1}\left(t-t_{0}\right)\right) \beta\left(\varepsilon^{-1}\left(s-s_{0}\right)\right)\right) a(t, s) d t d s=O_{\varepsilon}\left(\lambda^{-1}\right)
$$


Furthermore, if $\varepsilon>0$ is chosen so that $\partial_{t}^{2} \phi \neq 0$ on $\operatorname{supp} \beta\left(\varepsilon^{-1}\left(\cdot-t_{0}\right)\right) \beta\left(\varepsilon^{-1}\left(\cdot-s_{0}\right)\right)$, it follows from one-dimensional stationary phase that for each fixed $0 \leq s \leq 1$ we have

$$
\left|\beta\left(\varepsilon^{-1}\left(s-s_{0}\right)\right) \int_{-\infty}^{\infty} e^{i \lambda \phi(t, s)} \beta\left(\varepsilon^{-1}\left(t-t_{0}\right)\right) a(t, s) d t\right| \leq C \lambda^{-\frac{1}{2}},
$$

where $C$ is independent of $\varepsilon>0$ (cf. the proof of [10, Theorem 1.1.1]). This clearly implies that we have the uniform bounds $\left|I(\lambda)-I_{1}(\lambda)\right| \leq A \varepsilon \lambda^{-\frac{1}{2}}$ for all small $\varepsilon>0$. By combining this inequality with (2.13) we deduce that (2.12) holds in this case as well, which finishes the proof.

To use Lemmas 2.2 2.3, we also require another result which is essentially Lemma 3.1 in 4 .

Lemma 2.4. Given $\alpha \in \Gamma$ set

$$
\begin{aligned}
& K_{T, \lambda, \alpha}^{\gamma}(t, s) \\
& =\frac{1}{\pi} \int_{-\infty}^{\infty}(1-\beta(\tau)) \hat{\chi}(\tau / T) e^{-i \lambda \tau}\left(\cos \tau \sqrt{-\Delta_{\tilde{g}}}\right)(\tilde{\gamma}(t), \alpha(\tilde{\gamma}(s))) d \tau,|t|,|s| \leq 1 / 2 .
\end{aligned}
$$

Then if $\alpha \neq$ Identity and we set

$$
\phi_{\gamma, \alpha}(t, s)=d_{\tilde{g}}(\tilde{\gamma}(t), \alpha(\tilde{\gamma}(s))), \quad|t|,|s| \leq 1 / 2,
$$

then we can write for $|t|,|s| \leq 1 / 2$

$$
\begin{aligned}
K_{T, \lambda, \alpha}^{\gamma}(t, s)=\lambda^{\frac{1}{2}} w(\tilde{\gamma}(t), \alpha(\tilde{\gamma}(s))) \sum_{ \pm} a_{ \pm}\left(T, \lambda ; \phi_{\gamma, \alpha}(t, s)\right) e^{ \pm i \lambda \phi_{\gamma, \alpha}(t, s)} & \\
& +R_{T, \lambda, \alpha}^{\gamma}(t, s),
\end{aligned}
$$

where $w(x, y)$ is a smooth bounded function on $\mathbb{R}^{2} \times \mathbb{R}^{2}$ and where for each $j=0,1,2, \ldots$ there is a constant $C_{j}$ independent of $T, \lambda \geq 1$ so that

$$
\left|\partial_{r}^{j} a_{ \pm}(T, \lambda ; r)\right| \leq C_{j} r^{-\frac{1}{2}-j}, \quad r \geq 1
$$

and for a constant $C_{T}$ which is independent of $\gamma, \alpha$ and $\lambda$

$$
\left|R_{T, \lambda, \alpha}^{\gamma}(t, s)\right| \leq C_{T} \lambda^{-1}
$$

We shall postpone the proof of this result until the end and use it now, along with Lemmas 2.2 and 2.3, to prove (2.6). Recall that

$$
\begin{aligned}
&\left(\cos \tau \sqrt{-\Delta_{\tilde{g}}}\right)(\tilde{x}, \tilde{y})=0 \quad \text { if } d_{\tilde{g}}(\tilde{x}, \tilde{y})>|\tau| \\
& \text { and }\left(\cos \tau \sqrt{-\Delta_{\tilde{g}}}\right)(\tilde{x}, \tilde{y}) \text { is smooth if } d_{\tilde{g}}(x, y) \neq|\tau|
\end{aligned}
$$

Therefore since $\beta(\tau)=1$ for $|\tau| \leq 3$ we have $(1-\beta(\tau))\left(\cos \tau \sqrt{\Delta_{\tilde{g}}}\right)(\tilde{\gamma}(t), \tilde{\gamma}(s)) \in C^{\infty}$ if $0 \leq s, t \leq 1$, and so

$$
\int_{-T / 2}^{T / 2}(1-\beta(\tau)) \hat{\chi}(\tau / T) e^{-i \tau \lambda}\left(\cos \tau \sqrt{\Delta_{\tilde{g}}}\right)(\tilde{\gamma}(t), \tilde{\gamma}(s)) d \tau=O_{T}\left(\lambda^{-N}\right),
$$

for any $N=1,2,3, \ldots$. 
As a result, if we use (2.8), we conclude that we would have (2.6) if we could show that

$$
\begin{gathered}
\sum_{\alpha \in \Gamma \backslash \text { Identity }}\left|\iiint_{-T / 2}^{T / 2} b(t, s)(1-\beta(\tau)) \hat{\chi}(\tau / T) e^{-i \tau \lambda}\left(\cos \tau \sqrt{\Delta_{\tilde{g}}}\right)(\tilde{\gamma}(t), \alpha(\tilde{\gamma}(s))) d \tau d t d s\right| \\
\leq 1+C_{T} \lambda^{-\frac{1}{2}}
\end{gathered}
$$

By (2.19) there are are only finitely many nonzero summands here (actually $O(\exp (c T))$ ones). Consequently, we would have (2.20) if we could show that given $\varepsilon>0$ and Identity $\neq \alpha \in \Gamma$ we have

$$
\begin{array}{r}
\left|\iiint_{-T / 2}^{T / 2} b(t, s)(1-\beta(\tau)) \hat{\chi}(\tau / T) e^{-i \tau \lambda}\left(\cos \tau \sqrt{\Delta_{\tilde{g}}}\right)(\tilde{\gamma}(t), \alpha(\tilde{\gamma}(s))) d \tau d t d s\right| \\
\leq \varepsilon+C_{\alpha, \varepsilon} \lambda^{-\frac{1}{2}}
\end{array}
$$

Note that since $\tilde{\gamma}$ is a geodesic in $\left(\mathbb{R}^{2}, \tilde{\gamma}\right)$ and $\alpha$ is an isometry, it follows that $\alpha(\tilde{\gamma})$ is also a geodesic. It is a geodesic which is different from $\tilde{\gamma}$ if $\alpha$ is not in the stabilizer subgroup of $\Gamma$ of all deck transformations preserving $\tilde{\gamma}$. If our geodesic $\gamma$ in $(M, g)$ is not a periodic geodesic then the stabilizer subgroup is just the identity element. If $\gamma$ is a periodic geodesic in $M$ of minimal period $\ell>0$, then we must have $\ell \geq 10$, because of our assumption regarding the injectivity radius. In this case, every nontrivial element of the stabilizer group satisfies $\alpha(\tilde{\gamma}(s))=\tilde{\gamma}(s+k \ell)$ for some $k \in \mathbb{Z} \backslash 0$. By Lemma 2.4 for such a $\alpha$ with $k \neq 0$, modulo a term which is $O_{\alpha}\left(\lambda^{-1}\right)$ we have that the left side of (2.21) is equal to the sum over \pm of

$$
\lambda^{\frac{1}{2}}\left|\iint b(t, s) w(\tilde{\gamma}(t), \tilde{\gamma}(s+k \ell)) a_{ \pm}(T, \lambda ;|t-s-k \ell|) e^{ \pm i \lambda|t-s-k \ell|} d t d s\right|,
$$

with $a_{ \pm}$as in (2.17). Since $b \in C_{0}^{\infty}\left(\mathbb{R}^{2}\right)$ vanishes when $|t|$ or $|s|$ is larger than $\frac{1}{2}$ and $w \in C^{\infty}\left(\mathbb{R}^{2}\right)$, by a simple integration by parts argument this term is $O_{\alpha}\left(\lambda^{-\frac{1}{2}}\right)$, which means that we have (2.21) for all nontrivial elements of the stabilizer group of $\tilde{\gamma}$.

To prove that we also have (2.21) for the remaining case where $\alpha \in \Gamma$ is not in the stabilizer group, by the above, it is enough to show that, if $\phi_{\gamma, \alpha}(t, s)$ is as in (2.15), then given $\varepsilon>0$ there is a constant $C_{\alpha, \varepsilon}$ so that

$$
\begin{aligned}
\left|\iint b(t, s) w(\tilde{\gamma}(t), \alpha(\tilde{\gamma}(s))) a_{ \pm}\left(T, \lambda ; \phi_{\gamma, \alpha}(t, s)\right) e^{ \pm i \lambda \phi_{\gamma, \alpha}(t, s)} d t d s\right| & \\
& \leq \varepsilon \lambda^{-\frac{1}{2}}+C_{\alpha, \varepsilon} \lambda^{-1} .
\end{aligned}
$$

By Lemma 2.2 the phase function here either satisfies $\nabla_{t, s} \phi_{\gamma, \alpha} \neq 0$ on the support of the integrand, or there is a unique point $\left(t_{0}, s_{0}\right)$ in the support where $\nabla_{t, s} \phi_{\gamma, \alpha}$ vanishes and at that point $\frac{\partial \phi_{\gamma, \alpha}}{\partial t \partial s}\left(t_{0}, s_{0}\right) \neq 0$. In the former case by (2.11) the left side of (2.22) $O_{\alpha}\left(\lambda^{-1}\right)$, which is more than is required. In the latter case, we obtain the estimate from Lemma 2.3. Thus, we have established (2.22), which except for the proof of Lemma 2.4. completes the proof of Theorem 2.1 .

Proof of Lemma 2.4. Since the injectivity radius of $(M, g)$ is 10 or more, it follows that $d_{\tilde{g}}(\tilde{\gamma}(t), \alpha(\tilde{\gamma}(s))) \geq 10$ if $|t|,|s| \leq 1 / 2$. Also, as noted before, for each $T$, by Huygens principle, there are only finitely many terms in (2.14) that we must consider. 
Next, let for $x \in \mathbb{R}^{2},|x| \geq 1$,

$$
\begin{aligned}
K_{0}(|x|) & =\frac{1}{\pi} \int_{\mathbb{R}^{2}} \int_{-\infty}^{\infty} \hat{\chi}(\tau / T) e^{i \lambda \tau} \cos (\tau|\xi|) e^{i x \cdot \xi} d \tau d \xi \\
& =\int_{\mathbb{R}^{2}} T \chi(T(\lambda-|\xi|)) e^{i x \cdot \xi} d \xi+\int_{\mathbb{R}^{2}} T \chi(T(\lambda+|\xi|)) e^{i x \cdot \xi} d \xi .
\end{aligned}
$$

Also let $\Phi_{T}(\xi) \in \mathcal{S}(\mathbb{R})$ be defined by the Fourier transform $\hat{\Phi}_{T}(\tau)=\beta(\tau) \hat{\chi}(\tau / T)$ and put

$$
\begin{aligned}
K_{1}(|x|) & =\frac{1}{\pi} \int_{\mathbb{R}^{2}} \int_{-\infty}^{\infty} \beta(\tau) \hat{\chi}(\tau / T) e^{i \lambda \tau} \cos (\tau|\xi|) d \tau d \xi \\
& =\int_{\mathbb{R}^{2}} \Phi_{T}(\lambda-|\xi|) e^{i x \cdot \xi} d \xi+\int_{\mathbb{R}^{2}} \Phi_{T}(\lambda+|\xi|) e^{i x \cdot \xi} d \xi
\end{aligned}
$$

Recall that the Fourier transform of Lebesgue measure on the circle is of the form

$$
\widehat{d \theta}(y)=\int_{S^{1}} e^{i y \cdot(\cos \theta, \sin \theta)} d \theta=|y|^{-\frac{1}{2}} \sum_{ \pm} a_{ \pm}(|y|) e^{ \pm i|y|}, \quad|y| \geq 1,
$$

where for every $j=0,1,2, \ldots$ we have

$$
\left|\partial_{r}^{j} a_{ \pm}(r)\right| \leq C_{j} r^{-j}, \quad r \geq 1
$$

Also $\widehat{d \theta} \in C^{\infty}(\mathbb{R})$. Therefore, if $\lambda, T \geq 1$, modulo a term which is $O\left((\lambda|\xi|)^{-N}\right)$ for any $N$ independent of $T$, we have

$$
\begin{aligned}
K_{0}(|x|) & =|x|^{-\frac{1}{2}} \int_{0}^{\infty} T \chi(T(\lambda-r)) \sum_{ \pm} a_{ \pm}(|x| r) e^{ \pm i r|x|} r^{\frac{1}{2}} d r \\
& =\lambda^{\frac{1}{2}}|x|^{-\frac{1}{2}} \sum_{ \pm} b_{ \pm}(T, \lambda ;|x|) e^{ \pm i \lambda|x|}
\end{aligned}
$$

where one easily sees that

$$
\left|\partial_{r}^{j} b_{ \pm}(T, \lambda ; r)\right| \leq C_{j} r^{-j}, \quad r, \lambda, T \geq 1, j=0,1,2 \ldots
$$

Similar arguments show that, modulo an $O\left((\lambda|x|)^{-N}\right)$ error we also have

$$
K_{1}(|x|)=\lambda^{\frac{1}{2}}|x|^{-\frac{1}{2}} \sum_{ \pm} \tilde{b}_{ \pm}(T, \lambda ;|x|) e^{ \pm i \lambda|x|}
$$

where $\tilde{b}_{ \pm}$satisfy the bounds in (2.24).

To use this we shall use the Hadamard parametrix (see $\left[12\right.$ ). For $x, y \in \mathbb{R}^{2}$ we can write

$$
\begin{aligned}
\left(\cos \tau \sqrt{\Delta_{\tilde{g}}}\right)(x, y) & =(2 \pi)^{-2} w(x, y) \int_{\mathbb{R}^{2}} e^{i d_{\tilde{g}}(x, y) \xi_{1}} \cos (\tau|\xi|) d \xi \\
& +\sum_{ \pm} \int_{\mathbb{R}^{2}} e^{i d_{\tilde{g}}(x, y) \xi_{1}} e^{ \pm i \tau|\xi|} a_{ \pm}(\tau, x, y,|\xi|) d \xi+R(\tau, x, y)
\end{aligned}
$$

where we can take the remainder to satisfy

$$
|R(\tau, x, y)|+\left|\partial_{\tau} R(\tau, x, y)\right| \leq C_{T}, \quad \text { if }|\tau| \leq T,
$$

and $a_{ \pm}$is a symbol of order -2 which, in particular, satisfies

$$
\left|\partial_{\tau}^{j} a_{ \pm}(\tau, x, y,|\xi|)\right| \leq C_{T, j}(1+|\xi|)^{-2}, \quad \text { if }|\tau| \leq 2 T, j=0,1,2, \ldots,
$$

and where the leading coefficient $w$ is smooth, nonnegative and satisfies

$$
w(x, y) \leq C
$$


independent of $x, y \in \mathbb{R}^{2}$, by volume comparison theorems (see [1], [13]).

Clearly if we replace $\left(\cos \tau \sqrt{\Delta_{\tilde{g}}}\right)(\tilde{\gamma}(t), \alpha(\tilde{\gamma}(s)))$ by $R(\tau, \tilde{\gamma}(t), \alpha(\tilde{\gamma}(s)))$ in (2.14) we can integrate by parts in $\tau$ to see that the resulting expression satisfies the bounds in (2.18).

If we take $x=\tilde{\gamma}(t)$ and $y=\alpha(\tilde{\gamma}(s)),|t|,|s| \leq 1 / 2$ for the first term in the right side of (2.26) and replace the cosine-transform kernel in (2.14) by this expression then we will exactly obtain $(2 \pi)^{2}$ times

$$
w(\tilde{\gamma}(t), \alpha(\tilde{\gamma}(s))) K_{0}\left(\phi_{\gamma, \alpha}(t, s)\right)-w(\tilde{\gamma}(t), \alpha(\tilde{\gamma}(s))) K_{1}\left(\phi_{\gamma, \alpha}(t, s)\right),
$$

which can be taken as the first term in the right side of (2.16), with (2.17) being valid. Finally since (2.27) holds, the proof of this last assertion also shows that if in (2.14) we replace the cosine-transform kernel by the second term in the right side of (2.26) we obtain another term satisfying the bounds in (2.18) (in fact it is $O_{T}\left(\lambda^{-\frac{3}{2}}\right)$ ), which completes the proof.

\section{REFERENCES}

[1] P. H. Bérard, On the wave equation on a compact manifold without conjugate points, Math. Z. 155 (1977), 249-276.

[2] J. Bourgain, Geodesic restrictions and $L^{p}$-estimates for eigenfunctions of Riemannian surfaces, Linear and complex analysis, 27-35, Amer. Math. Soc. Tranl. Ser. 2, 226, Amer. Math. Soc., Providence, RI, 2009.

[3] N. Burq, P. Gérard and N. Tzvetkov, Restriction of the Laplace-Beltrami eigenfunctions to submanifolds, Duke Math. J. 138 (2007), 445-486.

[4] X. Chen and C. D. Sogge, A few endpoint geodesic restriction estimates for eigenfunctions, arXiv: 1210.7520

[5] M. Do Carmo, Riemannian geometry, Birkhäuser, Basel, Boston, Berlin, 1992.

[6] A. Good, Local analysis of Selberg's trace formula, Springer Lecture Notes 1040 (1983).

[7] D. Hejhal, Sur certaines séries de Dirichlet associées aux géodesiques fermées d'une surface de Riemann compacte, C. R. Acad. Sci. Paris 294 (1982), 273-276.

[8] A. Reznikov, Norms of geodesic restrictions for eigenfunctions on hyperbolic surfaces and representation theory, arXiv:math.AP/0403437

[9] A. Reznikov, A uniform bound for geodesic periods of eigenfunctions on hyperbolic surfaces, Forum Math., to appear.

[10] C. D. Sogge, Fourier integrals in classical analysis, Cambridge Tracts in Mathematics 105, Cambridge University Press, Cambridge, 1993.

[11] C. D. Sogge, Kakeya-Nikodym averages and $L^{p}$-norms of eigenfunctions, Tohoku Math. J. 63 (2011), 519-538.

[12] C. D. Sogge, Hangzhou lectures on eigenfunctions of the Laplacian, to appear in Annals of Math Studies, Princeton Univ. Press.

[13] C. D. Sogge and S. Zelditch, On eigenfunction restriction estimates and $L^{4}$-bounds for compact surfaces with nonpositive curvature, arXiv: 1108.2726

[14] S. Zelditch, Kuznecov sum formulae and Szegö limit formulae on manifolds, Comm. Partial Differential Equations 17 (1992), 221-260.

Department of Mathematics, Johns Hopkins University, Baltimore, MD 21218 